\title{
Agencia, mujeres y pintura: la experiencia de Débora Arango Pérez, 1950-1954*
}

\author{
Agency, women and painting: \\ the experience of Débora Arango Pérez, 1950-1954
}

\author{
Carolina Marrugo Orozco** \\ Universidad Andina Simón Bolívar, Quito, Ecuador.
}

Recibido: 16 de febrero de 2019. Aprobado: 16 de mayo de 2019.

DOI: $10.25100 /$ lamanzanadeladiscordia.v14i1.8062

Artículo de reflexión

\begin{abstract}
Resumen
Esta propuesta se inscribe en la línea de la Historia Cultural e historia de las mujeres. El objetivo es exponer una reflexión sobre la agencia de las mujeres en la pintura a mediados del siglo XX en Colombia (1950-1954), tomando como referente el caso de la pintora antioqueña Débora Arango Pérez. Este periodo coincide con su repliegue de la esfera pública y retiro a Casablanca. Sin embargo, deja ver que continuó siendo partícipe del campo artístico nacional y contribuyó como agente cultural en el contexto de la internacionalización de la producción artística. El corpus documental definido para este artículo incluye algunas fuentes de prensa (El Colombiano y Carpeta Débora Arango de la Sección de Artes y humanidades de la Biblioteca Luis Ángel Arango, Bogotá, Colombia).
\end{abstract}

Palabras clave: Mujeres; agencia; pintura, Débora Arango; siglo XX; historia de las mujeres.

\begin{abstract}
This proposal is part of the line of Cultural History and history of women. The objective is to present a reflection on the agency of women in painting in the mid-twentieth century in Colombia (1950-1954), taking as reference the case of the Antioquia painter Débora Arango Pérez. This period coincides with her withdrawal from the public sphere and retirement to Casablanca. However, it shows that she continued to be a participant in the national artistic field and contributed as a cultural agent in the context of the internationalization of artistic production. The documentary corpus defined for this article includes some press sources (El Colombiano and Carpeta Débora Arango of the Arts and Humanities Section of the Luis Ángel Arango Library, Bogotá, Colombia).
\end{abstract}

Keywords: Women; agency; painting; Débora Arango; twentieth century; women's history.

* El presente escrito es un artículo de reflexión producto de la investigación desarrollada por la autora en el marco de sus estudios de maestría entre 2017 y 2019, titulada “Con ojos de mujer: prácticas pictóricas y realidad política en Débora Arango Pérez, 1950-1954” financiada por la Universidad Andina Simón Bolívar- Sede Quito. Este trabajo se desarrolló bajo la dirección de la profesora Trinidad Pérez Arias, como requisito para optar al título de Magíster en Historia. El enfoque que privilegia este trabajo es el de la Historia de las mujeres, línea de investigación que la autora trabaja desde el año 2012. De manera más amplia, esta propuesta se articula a un interés por comprender la agencia de mujeres en el campo artístico, destacando el trabajo paralelo: "Cecilia Porras: la paleta vibrante del Caribe colombiano, con la cual fue ganadora de la Pasantía Nacional-Museo nacional de Colombia 2018 del Programa de Estímulos del Ministerio de Cultura.

** Historiadora y magíster en Historia por la Universidad Andina Simón Bolívar, Sede Quito, Ecuador. Docente e Investigadora independiente. Correo electrónico: krito981@hotmail.com. ORCID: 0000-0001-5165-9655 


\section{Introducción}

Como Débora Arango Pérez, muchas pintoras se destacaron en el contexto espacial y temporal de mediados del siglo XX en Colombia, aquellas encargadas de consolidar un espacio de larga data en las artes plásticas (Tabla 1). En el caso de esta pintora antioqueña, su participación deja evidencia de una agencia particular de las mujeres en la práctica pictórica moderna, específicamente en los espacios de creación estética de las formas realísticas, muy relacionadas con el expresionismo.

Tabla 1. Algunas mujeres pintoras a lo largo del siglo XX

\begin{tabular}{|c|c|c|c|}
\hline Nombre & Siglo & Técnica & Procedencia \\
\hline Albarracín de Barba, Josefina & $\mathrm{XX}$ & Escultora & Bogotá \\
\hline Arango Pérez, Débora & $\mathrm{XX}$ & Pintora & Medellín \\
\hline Álvarez de Castro, Astrid & $\mathrm{XX}$ & Pintora & Cartagena \\
\hline Arango Pérez, Débora & $\mathrm{XX}$ & Pintora & Medellín \\
\hline Balas, Irene & $\mathrm{XX}$ & Pintora & Budapest-Bogotá \\
\hline Ballesteros de Martínez, Gisela & $\mathrm{XX}$ & Pintora & Bogotá \\
\hline Biester Acevedo, Inés & $\mathrm{XX}$ & Retratista & París-Bogotá \\
\hline Bursztyn, Felisa & $\mathrm{XX}$ & Escultora & Bogotá \\
\hline Dávila de Trujillo, Sara & $\mathrm{XX}$ & Ceramista & Bogotá \\
\hline Daza, Beatriz & $\mathrm{XX}$ & Ceramista & Norte de Santander \\
\hline González de Cadena, Beatriz & $\mathrm{XX}$ & Ceramista & Barranquilla \\
\hline Holguín y Caro, Margarita & XIX & Pintora & Bogotá \\
\hline Lozano Ortiz, Margarita & $\mathrm{XX}$ & Pintora & París \\
\hline Márquez Montoya, Judith & $\mathrm{XX}$ & Pintora & Manizales \\
\hline Martín de Leudo, Mercedes & XIX & Pintora & Bogotá \\
\hline Merchán Fuquene, Helena & $\mathrm{XX}$ & Escultora & Boyacá \\
\hline Montaña de Izquierdo, Magdalena & XIX & Ceramista & Bogotá \\
\hline Negreiros, María Teresa & $\mathrm{XX}$ & Pintora & Brasil-Cali \\
\hline Ochoa Uribe, Mariela & $\mathrm{XX}$ & Pintora & Medellín \\
\hline Porras de Child, Cecilia & $\mathrm{XX}$ & Pintora & Cartagena \\
\hline Reyes, Emma & $\mathrm{XX}$ & Pintora & Bogotá \\
\hline Salgado, Graciela & $\mathrm{XX}$ & Pintora & Bogotá \\
\hline Sicard de Gómez, Inés & $\mathrm{XX}$ & Pintora, acuarelista & Bogotá \\
\hline Sinesterra de Carreño, Blanca & $\mathrm{XX}$ & Pintora, retratista & Bogotá \\
\hline Szalay de Wiedemann, Cristina & $\mathrm{XX}$ & Pintora & Polonia-Bogotá \\
\hline Tafur, Alicia & $\mathrm{XX}$ & Ceramista, escultora & Cali \\
\hline Tanco Cordovez de Gómez, Helena & $\mathrm{XX}$ & Pintora & Bogotá \\
\hline Tejada Sáenz, Lucy & $\mathrm{XX}$ & Pintora, muralista, grabadora & Pereira-Caldas \\
\hline Urrutia Holguín, Sofía & $\mathrm{XX}$ & Pintora, ceramista & Bolivia-Bogotá \\
\hline Vásquez de Arce y Bernal, Feliciana & XVII & Pintora, miniaturista & Bogotá \\
\hline Zarate, Nimia & $\mathrm{XX}$ & Pintora & Bogotá \\
\hline Zerda, María Teresa & $\mathrm{XX}$ & Escultora & Bogotá \\
\hline
\end{tabular}

Fuente: elaboración propia a partir de Ortega, Carmen (1979).

Nota: esta tabla permite visibilizar una presencia y participación activa, consecuente y variada de las mujeres en el ámbito de las artes plásticas a lo largo del siglo XX, junto a los lugares de procedencia que indica que el rango de práctica estuvo en el margen nacional e internacional. 
Al respecto, se puede decir que las mujeres participaron de la construcción del campo artístico desde mediados del siglo XIX en un espacio permisivo para ellas, puesto que no arriesgaba su lugar social. Después de todo, pintar, escribir, tejer y bordar fueron actividades surgidas en el espacio privado (Perrot, 2008). Empero, con el advenimiento de la modernidad y la influencia que impuso la burguesía urbana, se planteó una nueva posibilidad de que estas actividades se ampliaran a la esfera pública (Reyes, 1945). Estas formas artísticas como la pintura se situaron bajo nuevas formas de capital simbólico (Bourdieu, 1969, p. 97), especialmente entre las mujeres de la élite, quienes ampliaron su acceso a estos espacios.

Como una propuesta para visibilizar a las pintoras de mediados del siglo XX en Colombia, este trabajo se enfoca en exponer aspectos de la agencia de las mujeres pintoras, especialmente de la pintora antioqueña Débora Arango Pérez (1907-2005, Medellín, Colombia). Desde el punto de vista cultural, se pretende analizar, cómo factores de su entorno social -tales como educación y relaciones sociales de clase y género-, favorecieron su posicionamiento en el campo artístico colombiano y la mantuvieron dentro de la crítica de arte a pesar de su retiro voluntario en la década de 1950. A través de reportajes de prensa de El Colombiano y de la Carpeta Débora Arango de la Sección de Artes y humanidades de la Biblioteca Luis Ángel Arango (Bogotá, Colombia), se evidenciará su agencia particular durante mediados del siglo XX.

La corriente historiográfica que aborda esta perspectiva de estudio es la historia cultural, específicamente la que atiende a las mujeres como objeto de reflexión y sujetas históricas dotadas de una agencia. Más allá de constituirse en una estrategia "sumatoria," esta investigación busca comprender la forma en la que la pintora antioqueña formó parte del campo cultural colombiano en el contexto de mediados del siglo XX y logró posicionarse como una representante destacada del arte moderno en Colombia (Harding, 1987/1998).

Desde una perspectiva biográfica, se busca identificar a Débora Arango Pérez en relación con su pintura. En este sentido, se pretende comprender la materialidad del arte como un espacio de participación e interacción con el mundo y con los debates visuales, políticos y estéticos de mediados del siglo XX; y destacar su horizonte de reflexión en relación al arte como una práctica multifacética y abierta, enfocada en una narrativa interesada en las mujeres como vehículo hacia la crítica social (Levi, 1989).

Este trabajo consta de tres apartados. El primero se titula: "Débora Arango Pérez en perspectiva biográfica" en el cual se ubica la experiencia personal de la pintora con las circunstancias de su contexto social de mediados del siglo XX. La segunda parte se titula: "Débora Arango Pérez como agente cultural a mediados del siglo XX en Colombia" y plantea la agencia de la artista antioqueña a partir de una recepción desde la opinión pública expuesta en el periódico El Colombiano. Finalmente, el último apartado titulado "Retos y perspectivas en la historia de las mujeres pintoras en Colombia" expone los desafíos de comprender a las mujeres como agentes de la cultura nacional.

\section{Débora Arango Pérez en perspectiva biográfica}

Como género literario o propuesta historiográfica, la biografía nos permite establecer un acercamiento a la vida de los sujetos desde distintas perspectivas. El historiador italiano Giovanni Levi presenta en su trabajo "Los usos de la biografía" una posibilidad que permite reconocer, por ejemplo, de manera particular, aspectos de la agencia de la pintora Débora Arango Pérez entre 1950-1954 en Colombia. Dentro de las tipologías propuestas, este enfoque se refiere específicamente a la relación biografía y contexto, que permite analizar a una mujer artista inscrita en el contexto histórico y social de la Primera Violencia ${ }^{1}$ en Colombia, pero también como agente activa del campo cultural donde participaron otras mujeres con intereses similares (Levi, 1989).

El posicionamiento particular de una pintora como Débora Arango Pérez permite reconocer el perfil de una mujer artista a mediados del siglo $\mathrm{XX}$,

1 Según el investigador Orlando Fals este marco temporal corresponde al periodo que va de 1948 a 1957. Véase: Fals Orlando (1962). La violencia en Colombia (Tomo I). Bogotá: Taurus. 
cuando aún estas no gozaban de una ciudadanía legal. De hecho, este trabajo plantea que las mujeres en Colombia tuvieron una ciudadanía de facto $^{2}$, desarrollada en los espacios tradicionalmente permitidos y que se fueron abriendo nuevas posibilidades para ellas. Dichos espacios fueron pensados inicialmente para reforzar un "deber ser" a partir de la aprehensión de prácticas y hábitos asociados con el mundo, el arte, la escritura, la pintura, la práctica del viaje, etc., que se fueron convirtiendo paulatinamente en experiencias de autonomía.

Precisamente es a partir de experiencias cotidianas desde donde se pretende pensar la forma en que las mujeres como Débora Arango Pérez resignificaron escenarios como la pintura para establecer espacios autónomos, los cuales se derivaron de las inquietudes surgidas desde el proceso de creación artística. Esto es, la consecución de un proceso de creación continúo aunado al conjunto de oportunidades en el espacio público, desarrollando su agenciamiento en distintos ámbitos de las artes.

Obviamente, como lo expresa el historiador Eduardo Serrano (1995), el factor de clase fue importante para muchas mujeres pintoras en la consecución de ciertos objetivos, ya que permitió su articulación a los circuitos sociales, dominados hasta entonces por los hombres. Esto se dio principalmente por relaciones de consanguineidad y, más tarde, a partir de filiaciones académicas con reconocidos maestros formados en Europa de quienes habían recibido instrucción, y quienes establecieron la enseñanza artística como parte de programas de formación. Este aspecto no solamente da cuenta de una sucesión del conocimiento artístico sino de una especie de relación tutelar basada en el principio académico, pero también con tintes discipulares. En el caso de Débora Arango Pérez, sus experiencias cercanas fueron con los maestros Eladio Vélez y Pedro Nel Gómez, de los cuales admitió la base de su aprendizaje pictórico.

El factor de clase permite pensar que el lugar que la historia ha concedido a las mujeres en las distintas sociedades está permeado ante todo por su agencia, que como lo explica Sen (2000), se va expandiendo para ellas a medida que se abren nuevas oportunidades en distintos ámbitos como la educación y la promoción de derechos. En el caso de Arango, su inscripción temprana en los circuitos del arte, desde el ámbito familiar hasta la formación académica de la escuela primaria, le permitió ser parte de una experiencia significativa en el ámbito de las artes. A pesar de que su origen puede pensarse en el contexto conservador de la sociedad antioqueña, Débora fue parte de un contexto en el que el arte se constituyó en una de las pocas vías de libertad para las mujeres, como forma educativa y como una labor creativa, muy cercana al valor del trabajo. Aparte, la generación de Arango presenció un lugar activo de las mujeres como agentes sociales a través de las misiones, extendida en gran medida al ámbito cultural, en especial a lo relacionado con la apropiación de la identidad local (Melo, 1997). Ver Figura 1.

Estos intersticios permitieron a Arango y otras mujeres ser partícipes y testigo de la cotidianidad del siglo XX: un marco temporal lleno de contradicciones entre la modernización y la violencia (Bushnell, 1996). Esta experiencia amplió la presencia de mujeres en el espacio público, donde artistas como Débora Arango Pérez encontraron la materia prima para explotar su creatividad. Particularmente para la artista antioqueña, la extensión del ejercicio de creación generó nuevas inquietudes en el conocimiento autodidacta de saberes como la medicina. Aquí, el entorno familiar influyó para que la curiosidad de Débora quedara saciada con el material de su padre y hermano, ambos médicos (Galeano, 2004).

El temprano acceso al espacio público le confirió a Arango una forma de interacción con otras situaciones de la vida cotidiana como los bares, el mundo del trabajo, la maternidad, el desplazamiento y la violencia, entre otros. Estas variables fueron perceptibles a lo largo de su obra y retomaron experiencias de la realidad local y nacional como la prostitución, la migración rural, los escándalos políticos, etc. En el trabajo de la artista hubo una atención especial sobre las mujeres, enfocadas de manera particular en la exposición de las relaciones de poder y autoridad que estas experimentaron, el tabú de la sexualidad femenina y las experiencias

2 Se entenderá este concepto como la práctica ciudadana de las mujeres en la vida cotidiana, pese a no haber sido reconocidas legalmente. 


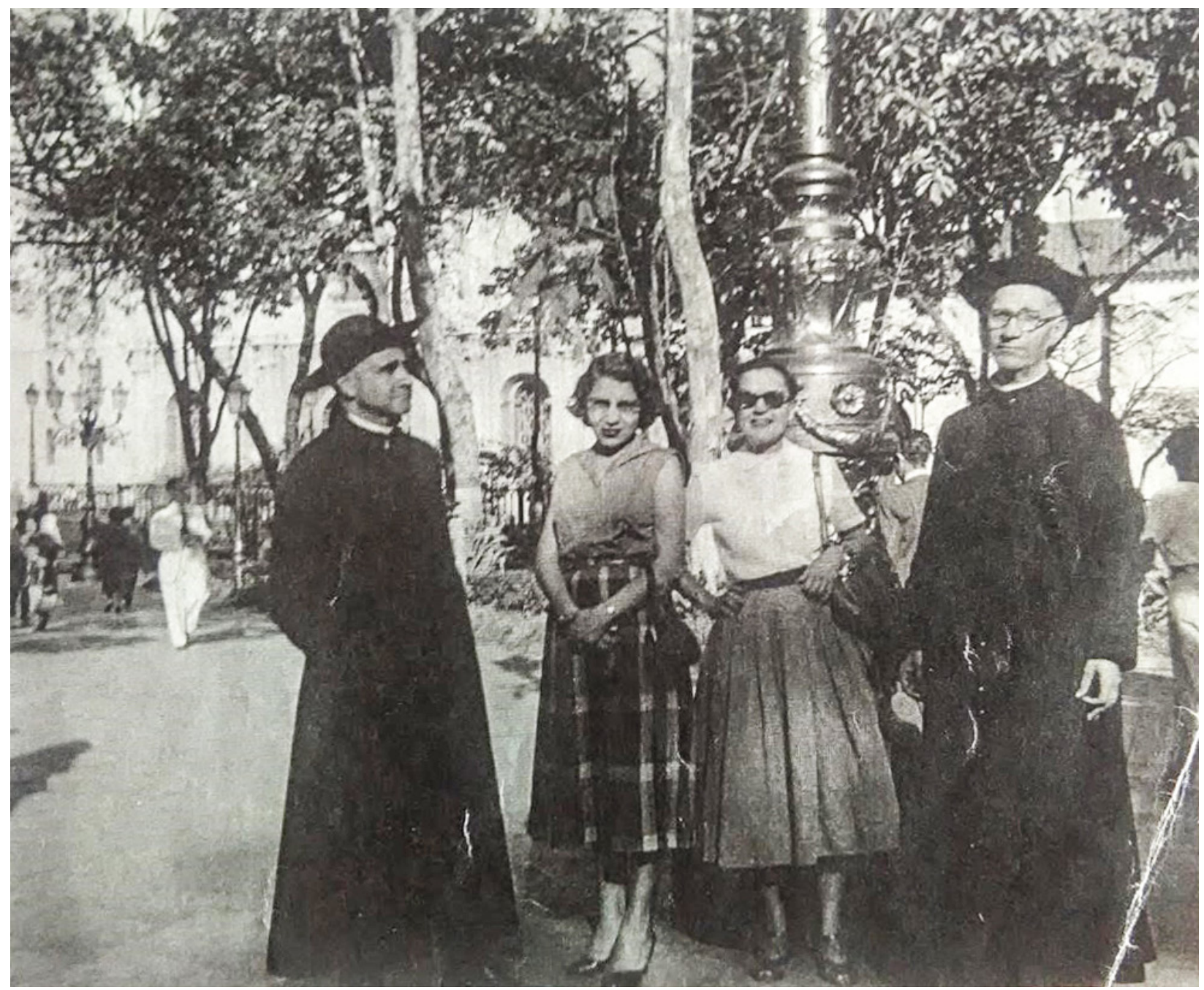

Figura 1. Fotografía Débora Arango Pérez

Fuente: Carpeta Débora Arango. Sección de Artes y humanidades Biblioteca Luis Ángel Arango. Bogotá. Sin registro de fecha al momento de consulta. ${ }^{3}$

tradicionales de las mujeres como la maternidad, inscritas en narrativas del conflicto. Esta intensa curiosidad deja ver a la mujer artista en constante diálogo con sus experiencias cotidianas. Dicha evidencia de asimilación de estos saberes permite identificar la presencia de un criterio y una conciencia de decisión de Débora dentro de un campo de disputas sociales, políticas, económicas y culturales que atravesaban la realidad de las mujeres (Scott, 1996). Esta experiencia se asemeja en gran parte a la de otras mujeres artistas, quienes reconocen en la familia y la educación religiosa dos pilares de la formación artística.

El mundo de las artes fue uno de los primeros espacios de amplia participación de las mujeres, sin desconocer que en él también existieron marcadas tendencias de género y una especie de división sexual del arte, plasmada en el tipo de producción que estaba estipulada para cada uno (Londoño, 1995). Al respecto, los salones de arte regionales y nacionales, iones de larga data, sirvieron de espacio de formación e interacción, dando mayor sentido de

3 Esta fotografía fue consultada en la Carpeta Débora Arango, que contiene 50 documentos, muchos de ellos sin referencias precisas de fecha, entre las cuales se encuentra este material. Cabe aclarar que esta información puede ser verificada en la Colección Archivo de Artes de la sala de artes y humanidades de la Biblioteca Luis Ángel Arango en la ciudad de Bogotá. 
valoración para las mujeres y ampliando el horizonte de su participación. Así mismo, este mundo permitió una mayor cualificación del trabajo artístico y de una práctica de la competencia, mediada también por la presencia activa de la opinión de otras mujeres destacadas en el campo de las artes y las letras como Judith Márquez y Marta Traba.

La forma de desnaturalizar una visión sobre las mujeres se realizó por medio de la puesta en escena de una narrativa pictórica propositiva. La pintura de Arango es precisamente una invitación a desafiar el formato tradicional de la pintura, a ser testigos de las formas agresivas del color, a presenciar el carácter humano y natural de las mujeres, a desafiar los tabúes del cuerpo y a confrontar nuevas visiones de poder entre distintos actores sociales. Su pintura de mediados del siglo XX permite ver la confluencia de la realidad que vivían las mujeres con el diálogo de sus imaginarios. La pictórica de Arango remite a personajes de la literatura, al mundo místico, de la intelectualidad y de las formas tradicionales de "ser mujer," interpelando sus discursos contemporáneos como el de la "maternidad patriótica"4 (Luna y Villarreal, 1994). Su propuesta se enmarcó en un diálogo con la tradición pictórica de las mujeres para entrar a controvertirla y sugerirla desde distintos ámbitos, en especial, enfocado a un desafío de los tabúes sobre el cuerpo de las mujeres. Estas nuevas formas del arte estuvieron alejadas de las connotaciones mítico-religiosas bajo la cual se habían construido las representaciones tradicionales de las mujeres.

Al aplicar una desacralización a las formas pictóricas, logra incorporar en el ojo público nuevas formas estéticas que desafiaron el "deber ser," principalmente en ese contexto donde las mujeres adquirieron mayor visibilidad a través de la publicidad que creó imágenes idealizantes y se construyeron representaciones de la feminidad asociadas a la estética del consumo. El carácter testimonial de la obra de Arango radica entonces en esta propuesta de la mirada sensible de los actores sociales en el contexto de censura que vivía el país. Al pensar, por ejemplo el significado de esta coyuntura en nuestro presente, nos hace valorar aún más la función testimonial que adquiere la pintura como forma de producción cultural de una época (Archila, 1995).

\section{Débora Arango Pérez como agente cultural a mediados del siglo XX en Colombia}

E1 23 de julio de 1950, en la Sección "Nuestros valores" del periódico conservador El Colombiano, el periodista Ernesto Barrientos (1950), en el espacio periodístico de Suplemento, tituló una entrevista hecha a Débora Arango Pérez "Realidad social en la pintura de Débora Arango". En ella referenció que "la pintora antioqueña representaba la más libre expresión de las vanguardias del país" (p. 2). También se refirió al posicionamiento de Arango en relación a sus contemporáneos: "su maestro Pedro Nel Gómez y compañeros Carlos Correa, Rafael Sánchez y Gabriel Posada Zuluaga, de los cuales se distinguió por su vigorosa actitud frente a los comentarios sobre su obra” (p. 2). El periodista también destacó la cronología que quedó plasmada en sus óleos y acuarelas. Ver Figura 2.

Un año más tarde, en la sección de notas culturales del periódico El Colombiano del 20 de agosto de 1950, se publicó otra referencia aludiendo a su actividad artística:

próximamente viajará a Europa la consagrada pintora antioqueña Débora Arango, uno de los grandes valores que en el campo de las artes plásticas, posee Colombia. Silenciosamente ha laborado Débora durante los últimos años y sus obras han merecido el elogioso comentario de destacados críticos colombianos y europeos que han tenido el privilegio de conocerlos. Por las conocidas dificultades de nuestro estrecho ambiente las maravillosas pinturas de Débora Arango no han sido exhibidas en público. En España seguirá un curso especial en una de las mejores academias y aprovechará el ofrecimiento para exponer en las salas de la península y de los principales países de Europa. (Notas culturales, 1950, p. 5)

Las dos noticias dejan clara una presencia de Débora Arango Pérez en el campo artístico de Colombia a mediados del siglo XX, a pesar de que esta etapa coincide con su repliegue de la escena pública.

4 La maternidad patriótica se comprendió como el imaginario que acotó el acceso a la ciudadanía de las mujeres, a partir de la idea de "buena madre." 


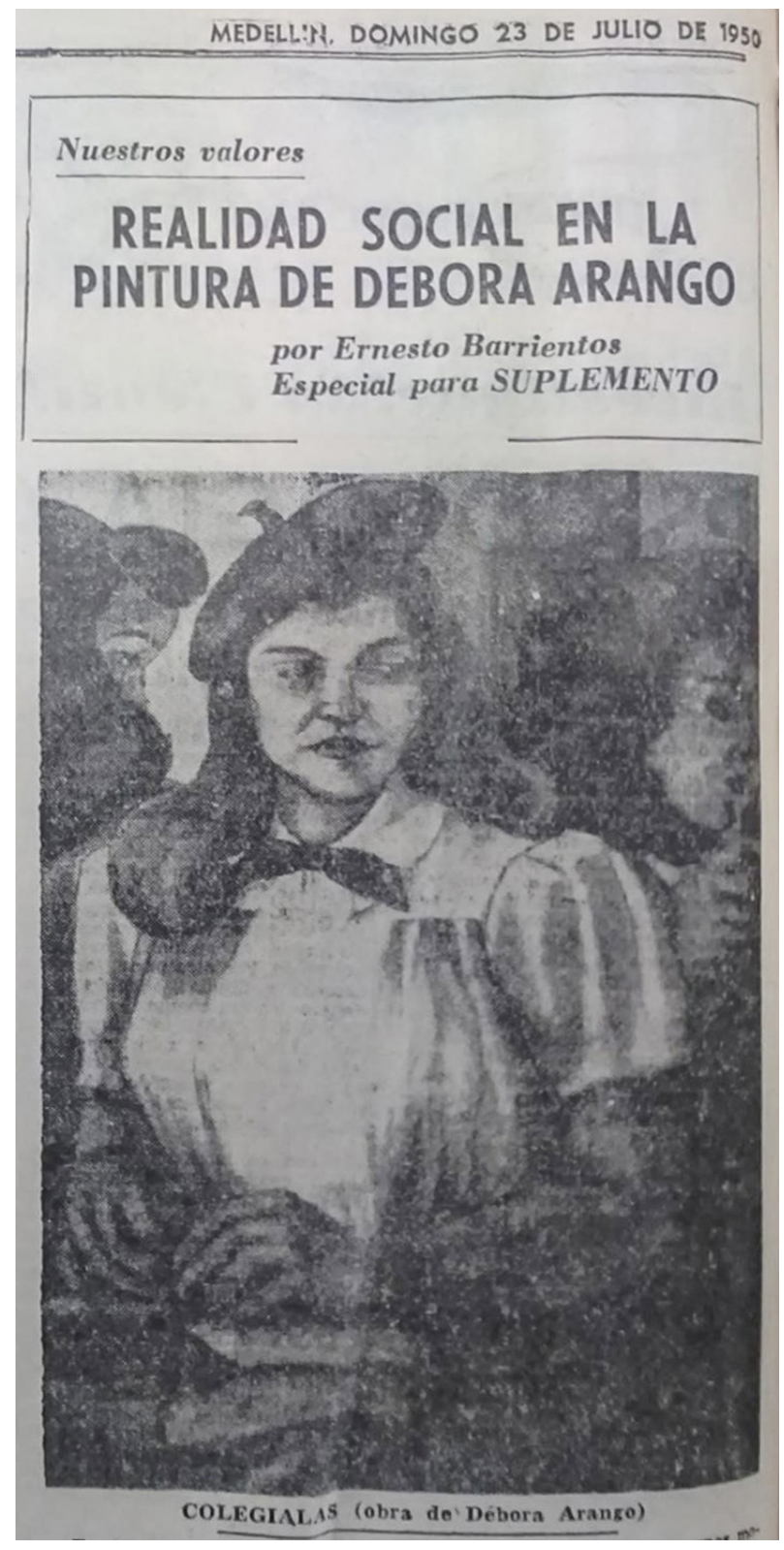

Figura 2. Realidad social en la pintura de Débora Arango

Fuente: periódico El Colombiano, 1950

Pero además, deja ver tres aspectos importantes a destacar: en primer lugar, el posicionamiento de su producción artística como parte de un estilo estético asociado con las Vanguardias. Hay que recordar que, a mediados del siglo XX en el contexto de la posguerra, esta propuesta artística como movimiento estuvo muy presente en el orden estético occidental. (Casas, 2006, p. 1). Según el historiador Álvaro Medina (2013), este movimiento fue lento en Colombia a diferencia de otros países de América Latina, por lo cual habría que valorar que la agencia de Débora Arango Pérez estuvo presente al mantener este estilo.

En segundo lugar, permite ubicar las obras de Arango en un diálogo con la producción nacional del campo artístico. Esto nos remite necesariamente a la idea de integración, debates, tensiones, acuerdos y desacuerdos en el campo de reglas en instituciones presentes en el circuito del arte. Lo anterior es importante destacarlo porque permite comprender que a pesar de que en esta época estuvieron en boga las formas abstractas y no figurativas con Cecilia Porras, Judith Márquez, Emma Reyes, Lucy Tejada, entre otras, las formas realísticas como las de Débora Arango Pérez se mantuvieron en interacción con la de otras mujeres.

En tercer lugar, la artista antioqueña queda totalmente relacionada con sus pares contemporáneos, hombres -algunas veces desde la relación tutelar de maestro-alumno-, lo cual permite una relación contigua y necesaria para fortalecer las experiencias del campo cultural. Ver Figura 3.

Como situación particular, durante la mitad del siglo XX hubo un protagonismo del pensamiento de las mujeres, especialmente porque estas construyeron una trayectoria en el campo de la formación literaria, artística e intelectual como cronistas, críticas de arte, gestoras culturales, pintoras, etc. Se destacó aquí una agencia que pasó de las ideas a la acción, a través de una autoconciencia individual de su labor como sujetos sociales y políticos.

Hay que recordar que el fortalecimiento de este campo se vio favorecido por la producción, circulación, difusión de conocimiento y prácticas artísticas. ${ }^{5}$ Las principales ciudades del país como Bogotá y Medellín se convirtieron en escenarios de difusión

5 A partir de la segunda mitad del siglo XX se abre un espacio de reflexión a través de las revistas especializadas en arte y literatura, desde las cuales es posible rastrear la existencia de una preocupación particular por la pintura. El registro documental consultado deja ver comentarios de especialistas en el arte como Clemente Airó, Marta Traba, Casimiro Eiger, Judith Márquez y Walter Engel, quienes participaron como críticos en reportajes que aludieron a aperturas de galerías, escritura de libros de arte, comentarios de obras de artistas, etc. Esto denota no sólo la expansión del campo artístico en Colombia, sino la permanente colaboración coadyuvada por la agencia de los medios impresos especializados. Para mayor ampliación véase: Espiral. Revista mensual de artes y letras (1949-1953). Plástica. Revista de arte contemporáneo 1955. 


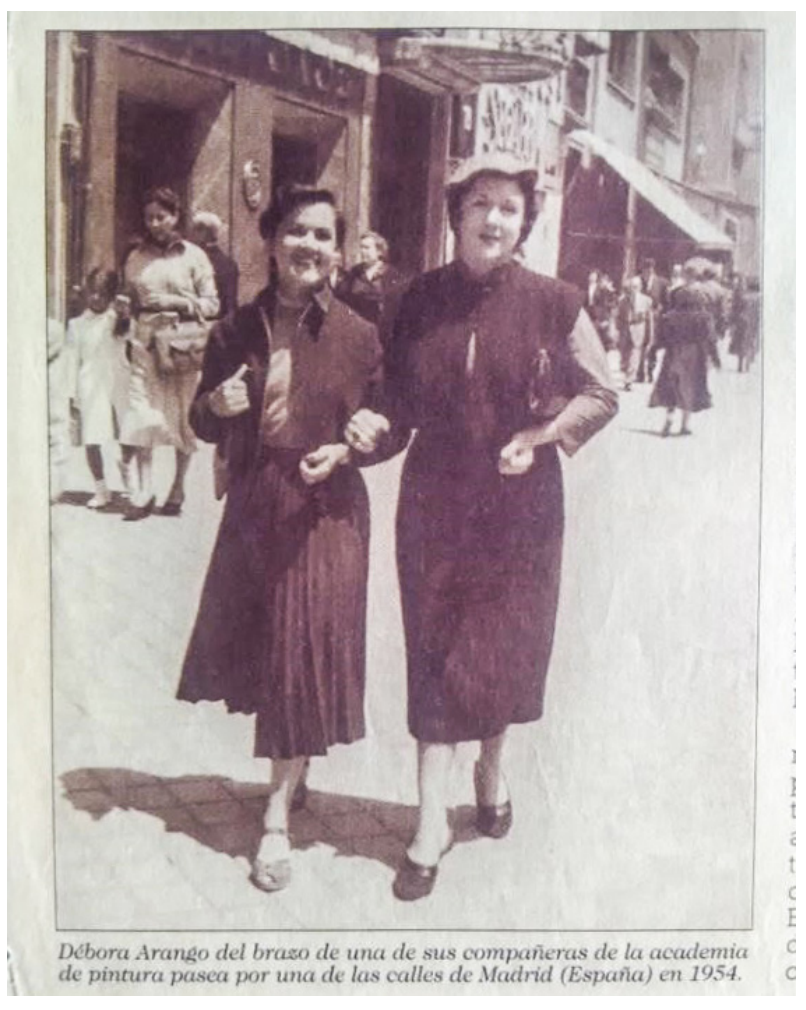

Figura 3. Débora Arango del brazo de una de sus compañeras de la academia de pintura pasea por una de las calles de Madrid (España) en 1954

Fuente: Revista Carrusel pág. 7. Carpeta Débora Arango. Colección Archivo de Artes de la sala de artes y humanidades de la Biblioteca Luis Ángel Arango en Bogotá.

del arte con la inauguración de galerías, exposiciones nacionales e internacionales y los salones de artistas que las mujeres aprovecharon. Este último espacio se expresó como una muestra de la integración y agremiación regional que experimentaban los pintores del país y en el cual se articulaban ejes de reflexión sobre los estilos pictóricos, hecho que se contrapone a una idea de desarticulación regional en Colombia (Londoño, 2005). En todo caso, este contexto permite evidenciar que la experiencia cultural en Colombia logró una integración significativa de las regiones.

Entre 1950 y 1954, Colombia ya experimentaba una expansión "hacia afuera" de las artes plásticas: un proceso de internacionalización mediado por la intensa presencia y agencia de mujeres y hombres artistas que se vincularon de manera activa al mundo de la plástica. En este contexto, el campo cultural vivió importantes contrastes, pues por un lado se habían posicionado con gran fuerza los llamados
"Abstractos" o artistas inclinados hacia las formas no-figurativas como Alejandro Obregón, Enrique Grau, Guillermo Wiedemann y Eduardo Ramírez Villamizar. Y por el otro, se empezaron a consolidar nuevos talentos como Débora Arango Pérez, Cecilia Porras de Child, Emma Reyes, Lucy Tejada, entre otras, las cuales coincidieron en un interés por posicionar la plástica como un espacio de trabajo reconocido para las mujeres.

\section{Retos y perspectivas en la historia de las mujeres pintoras en Colombia}

En este panorama incompleto de la producción pictórica de Débora Arango Pérez se intentó evidenciar una presencia activa de las mujeres en el campo artístico de mediados del siglo XX en Colombia. Esta antioqueña forjó una identidad pictórica, en esencia, reconocida por sus pares artistas, como parte de un movimiento que se inclinó por el arte realístico de inspiración europea y mexicana.

Las trayectorias de su producción comprueban que se instaló en los circuitos del arte local, nacional e internacional al lado de artistas reconocidos como Lucy Tejada, Emma Reyes, Sofía Urrutia, Alejandro Obregón, Enrique Grau, etc. Su contexto estuvo habitado por mujeres consolidadas en el mundo cultural e intelectual como Marta Traba, Meira del Mar y Judith Márquez, entre otras, desde las cuales es posible reconocer que la agencia cultural de las mujeres se movió en distintos ámbitos en la cultura nacional.

Débora Arango Pérez fue hija, hermana, alumna, artista, maestra. Fue rebelde, autónoma, arriesgada y poeta. Su pintura expresó ante todo el valor de la libertad y una visión diferente del mundo de las mujeres, la cual definió desde características multifacéticas.

Historiar la agencia de las mujeres pintoras es una tarea que apenas comienza. Visibilizarlas permitirá comprender de forma creciente el papel significativo que han jugado como agentes de la cultura nacional y reconocer a través de ellas, las trayectorias que experimentó el campo artístico. Permitirá conocerlas y reconocer sus posibilidades como agentes históricos por medio de sus experiencias particulares. 
Esta reflexión permite un acercamiento al diálogo que conservan las mujeres artistas como Débora Arango Pérez con los imaginarios tradicionales y la forma en que ellas deciden ser influidas o seducidas por nuevos estilos del arte. Significa pensar también en las formas que estas mujeres han aprehendido la pintura como práctica profesional, y su interacción con las instituciones de poder en el campo cultural que ejercen influencia en el ámbito artístico en el que operan. Así mismo, permite indagar sobre las diversas formas de expresión de las que hacen uso y el carácter personal que les aplican: la manera en sí que se apropian de la pintura como evidencia de una agencia.

De igual manera, la reflexión sobre la trayectoria de Débora Arango Pérez permite evidenciar dos aspectos importantes: por un lado, su incuestionable contribución al campo de las artes a lo largo del siglo $\mathrm{XX}$, lo cual deja una herencia incalculable para la tradición pictórica nacional. Por el otro, a través de la producción de esta artista se logra incorporar a la pintura como depósito material y testigo de la presencia de las mujeres en la historia: como una forma en que ellas producen una obra, al tiempo en que son representadas por otro ojo femenino (Scott, 1991).

Esta reflexión intentó explorar la presencia de Débora Arango en un marco temporal poco indagado por la historiografía tradicional (1950-1954). Durante este periodo se evidencia que la artista forma parte activa de los procesos de construcción de la cultura nacional y que en esas búsquedas, presencias y trayectorias, es capaz de sobrepasar los márgenes de su canon tradicional. Así mismo, permite identificar que es una sujeta creadora y promotora de la cultura en una sociedad que puede sobrepasar la acción de la sospecha para ejercer su autonomía y criterio de creación.

Aunque indefinible, la producción de Arango queda atravesada por un elemento común: la historia de las mujeres. Al evidenciar esta sensibilidad especial se hace interesante pensar en la artista que puede reflexionar sobre su género, a partir de otras experiencias y otras miradas. El posicionamiento en la forma en la que observa las experiencias de las mujeres puede llevar a reconocer un punto de interés muy útil para la posteridad: una fuente documental para la historia de las mujeres.

\section{Referencias bibliográficas}

Archila, Mauricio. (1995). Protestas sociales en Colombia 1946-1958. Historia Crítica, (11), 63-78.

Bourdieu, Pierre. (1969). Campo intelectual y proyecto creador. México: Siglo veintiuno editores.

Bushnell, David. (1996). Colombia una nación a pesar de sí misma. Bogotá, Colombia: Planeta.

Casas, Jorge. (2006). Nacionalismo y bachuismo en las esculturas boyacenses. Revista Educación y Ciencia, (9), 67-72.

Fals, Orlando. (1962). La violencia en Colombia (Tomo I). Bogotá, Colombia: Taurus.

Galeano, Ángel. (2004). Débora Arango. El arte, venganza sublime. Bogotá, Colombia: Panamericana.

Harding, Sandra. (1998). ¿Existe un método feminista? En: E. Bartra (Comp.). Debates en torno a una metodología feminista, (pp.9-34). México DF: PUEG, UAM. (Obra original publicada en 1987)

Levi, Giovanni. (1989). Los usos históricos de la biografía. Annales ESC, (6), 1335-1336.

Londoño, Santiago. (1995). Las mujeres y el arte en Colombia. En Magdala Velásquez (Ed.), Las mujeres en la historia de Colombia. Mujeres y Cultura Vol. III, (pp. 274-301). Bogotá, Colombia: Norma.

Londoño, Santiago. (2005). Breve historia de la pintura en Colombia. Bogotá, Colombia: Fondo de Cultura Económica.

Luna, Lola., y Villarreal, Norma. (1994). Historia, género y política. Movimientos de mujeres y participación política en Colombia, 1930-1991. Barcelona, España: Seminario Interdisciplinar Mujeres.

Medina, Álvaro. (2013). La renovación vanguardista, 19101950. Guía de estudio $N^{\circ} 135$. Bogotá, Colombia: Banco de la República. Recuperado de: http://www.banrepcultural.org/coleccion-de-arte-banco-de-la-republica/ curaduria/la-renovaci\% $\% 3 \%$ B3n-vanguardista.

Melo, Jorge. (1997). Espacio e Historia en Medellín [Mensaje en un blog: Colombia es un tema]. Recuperado de http://www.jorgeorlandomelo.com/thistoant.html.

Ortega, Carmen. (1979). Diccionario de artistas en Colombia. Bogotá, Colombia: Plaza \& Janes Editores Colombia Ltd.

Perrot, Michel. (2008). Mi historia de las mujeres. Buenos Aires, Argentina: Fondo de Cultura Económica.

Reyes, Ana. (1945). Cambios en la vida femenina durante la primera mitad del siglo XX. Colombia Credencial Historia, (68), 1-20.

Scott, Joan. (1991). The Evidence of Experience. Critical Inquiry, 17(4), 773-797. 
Scott, Joan. (1996). El género: Una categoría útil para el análisis histórico. En Marta Lamas (Comp.), El género: la construcción cultural de la diferencia sexual (pp. 265-302). México: PUEG.

Sen, Amartya. (2000). Desarrollo y libertad. Buenos Aires, Argentina: Planeta.

Serrano, Eduardo. (1995). Las mujeres y el arte en Colombia. En Magdala Velásquez (Ed.), Las mujeres en la historia de Colombia. Mujeres y Cultura Vol. Tomo III (pp. 256-273). Bogotá, Colombia: Norma.

\section{Fuentes primarias}

Barrientos, Ernesto. (23 de julio de 1950). Realidad social en la pintura de Débora Arango. El Colombiano, p. 2.

Carpeta Débora Arango. Colección Archivo de Artes de la sala de artes y humanidades. Bogotá, Colombia: Biblioteca Luis Ángel Arango.

Notas culturales. (20 de agosto de 1950). El Colombiano, p. 5. 